\title{
Interplay between IFN- $\gamma$ and IL-6 signaling governs neutrophil trafficking and apoptosis during acute inflammation
}

\author{
Rachel M. McLoughlin, ${ }^{1,2}$ Janusz Witowski, ${ }^{1}$ Rachel L. Robson, ${ }^{1}$ Thomas S. Wilkinson, ${ }^{1}$ \\ Suzanne M. Hurst, ${ }^{2}$ Anwen S. Williams, ${ }^{3}$ John D. Williams, ${ }^{1}$ Stefan Rose-John, ${ }^{4}$ \\ Simon A. Jones, ${ }^{2}$ and Nicholas Topley ${ }^{1}$ \\ ${ }^{1}$ Institute of Nephrology, University of Wales College of Medicine, Cardiff, United Kingdom \\ ${ }^{2}$ Cardiff School of Biosciences, Molecular Cell Biology Research Group, Cardiff University, Cardiff, United Kingdom \\ ${ }^{3}$ Rheumatology Research Laboratories, University of Wales College of Medicine, Cardiff, United Kingdom \\ ${ }^{4}$ Kiel Institut für Biochemie, Christian-Albrechts-Universität zu Kiel, Kiel, Germany
}

Regulated recruitment and clearance of neutrophils (PMN) is the hallmark of competent host defense and resolution of inflammation. We now report that IFN- $\gamma$ controls PMN infiltration and modulates IL-6 signaling through its soluble receptor (sIL-6R) to promote their apoptosis and clearance. Induction of peritoneal inflammation in IFN- $\gamma$-deficient $\left(I F N-\gamma^{--}\right)$mice emphasized that the initial rate of PMN recruitment was impaired. This defect in PMN recruitment was also associated with the suppressed intraperitoneal expression of IL- $1 \beta$ and IL- 6 . Reconstitution of IFN- $\gamma$ signaling restored the rate of PMN infiltration and IL-6 levels and was accompanied by normalization of PMN-activating CXC chemokine expression. To test whether local IL- 6 signaling modulated PMN recruitment, inflammation was induced in $I F N-\gamma^{-/}$and $I L-6^{-/-}$mice and cytokine signaling adapted by intraperitoneal sIL-6R-IL-6 fusion protein (HYPER-IL-6) or IFN- $\gamma$. Although HYPER-IL-6 attenuated PMN influx in $I F N-\gamma^{-/}$mice, IFN- $\gamma$ had no effect on PMN infiltration in $I L-6^{-/-}$mice. Examination of the leukocyte infiltrate from $I F N-\gamma^{-1}, I L-6^{-/-}$, and wild-type mice showed that apoptosis was aberrant in the absence of IFN- $\gamma$ and IL- 6 as a result of impaired sIL-6R signaling. These data emphasize a pivotal role for IFN- $\gamma$ in regulating innate immunity through control of both the recruitment and clearance phases of PMN trafficking.

J. Clin. Invest. 112:598-607 (2003). doi:10.1172/JCI200317129.

\section{Introduction}

Although IFN- $\gamma$ plays a central role in controlling the host's response to bacterial and viral infection, the precise cellular mechanisms that contribute to this obligate role in host defense have not been fully elucidated (1). These IFN- $\gamma$-dependent events depend exclusively on signal transducer and activator of transcription factor 1 (STAT1) activation, since mice lacking this signal transducer are defective in their ability to resolve bacterial and viral infections (2-4). That IFN- $\gamma$ is important in regulating host defense is emphasized by evidence of its effect on leukocyte recruitment. In this respect, IFN- $\gamma$

Received for publication October 14, 2002, and accepted in revised form June 3, 2003.

Address correspondence to: Nicholas Topley, Institute of Nephrology, University of Wales College of Medicine, Heath Park, Cardiff, CF14 4XN, United Kingdom. Phone: 44-29-20748432; Fax: 44-29-20748470; E-mail: topley@cf.ac.uk.

Conflict of interest: The authors have declared that no conflict of interest exists.

Nonstandard abbreviations used: signal transducer and activator of transcription factor 1 (STAT1); neutrophils (PMN); soluble IL-6 receptor (sIL-6R); mononuclear cells (MNC); soluble gP130 (sgp130); human peritoneal mesothelial cells (HPMC); digoxigenin (DIG); electrophoretic mobility shift assays (EMSA); S. epidermidis cell-free supernatant (SES); propidium iodide (PI); p-nitroaniline (pNA). can both enhance and suppress chemokine secretion in response to proinflammatory cytokines (IL-1 $\beta$ and TNF- $\alpha$ ), modulate chemokine receptor expression, and affect cellular adhesion and transmigration (5-10). In vivo, however, it is unclear whether these actions on leukocyte trafficking are a direct result of IFN- $\gamma$ or occur through its modulation of other mediator cascades during the inflammatory response. Recent data, however, suggest that the initial activation of resident $\mathrm{CD} 4 \mathrm{~T}$ cells to produce IFN- $\gamma$ (and IL-17) are important in directing neutrophil (PMN) recruitment and in resolving bacterial infection (11-13).

During acute inflammation, PMN trafficking is tightly regulated to provide effective host defense without promoting tissue injury. Initially, PMN are recruited from the circulation toward sites of inflammatory insult or infection in response to local secretion of neutrophil-activating chemokines containing the ELR amino acid motif (ELR ${ }^{+}$(14). As inflammation proceeds, or infection subsides, PMN are removed in a controlled manner to prevent persistent tissue necrosis (15). The initial phase of PMN clearance results from the downregulation of local $\mathrm{ELR}^{+} \mathrm{CXC}$ chemokine expression and a switch to other chemokines selective for the attraction of mononuclear cells (MNC) (16). Central to the removal of PMN is the 
process of programmed cell death (apoptosis), which renders the cells susceptible to nonphlogistic phagocytosis by the MNC infiltrate. The controlled process of PMN trafficking thus provides an effective mechanism for limiting inflammation-induced tissue damage. Ultimately, this leads to clearance of infection and resolution of inflammation $(13,15,17)$. Consequently, the pattern of PMN infiltration encountered during an inflammatory episode is defined by the temporal and differential regulation of chemokines and PMN apoptosis.

To define the regulatory events that dictate the pattern of leukocyte trafficking encountered during inflammation, we have used an established murine model of acute peritoneal inflammation using either a cell-free supernatant derived from Staphylococcus epidermidis or IL- $1 \beta$ as a stimulus $(8,16)$. This model has allowed the examination of the temporal changes in leukocyte infiltration and inflammatory and chemotactic mediator expression. Changes in this model closely resemble those observed in human bacterial peritonitis characterized by temporal changes in the expression patterns of both proinflammatory and chemotactic cytokines, the rapid recruitment of PMN, and their subsequent replacement by a more sustained influx of MNC (16). Although the factors that orchestrate resolution of acute inflammation and restoration of normal tissue architecture are not fully defined, we have recently characterized important roles for both IL-6, through its soluble IL- 6 receptor (sIL-6R), and IFN- $\gamma$ signaling in regulating the transition between PMN and MNC infiltration $(8,16)$. In the present study, we extend these observations and have identified that IFN- $\gamma$ dictates the pattern of PMN recruitment encountered by affecting the local activities of IL-1 $\beta$ and IL-6. In this way, IFN- $\gamma$ is able to tightly control the initial recruitment of PMN and promote their subsequent clearance to ensure effective host defense and resolution of inflammation.

\section{Methods}

Reagents. All chemicals were purchased from SigmaAldrich (St. Louis, Missouri, USA) unless otherwise stated. Recombinant human IL- $1 \beta$, TNF- $\alpha$, IFN- $\gamma$ $\left(10^{7} \mathrm{U} / \mathrm{mg}\right.$ of protein), and soluble gp130 (sgp130) were from R\&D Systems (Abingdon, United Kingdom). Recombinant murine IL- $1 \beta$ and IFN- $\gamma$ were from Peprotech Ltd. (London, United Kingdom). Endotoxin levels in recombinant cytokines were less than $0.1 \mathrm{ng} / \mu \mathrm{g}(1 \mathrm{EU} / \mu \mathrm{g})$ of protein. Tissue culture reagents were from GibcoBRL/Life Technologies (Paisley, United Kingdom), and all culture flasks and multiwell plates were obtained from Falcon (BD Biosciences, Oxford, United Kingdom). PBS ( $\mathrm{pH}$ 7.4) was obtained from GibcoBRL/Life Technologies. HYPER-IL-6 was prepared as previously described (18) and stored at $-70^{\circ} \mathrm{C}$. A fresh aliquot was used for each experiment.
Isolation and culture of human peritoneal mesothelial cells. Human peritoneal mesothelial cells (HPMC) were isolated by tryptic digestion of greater omental tissue obtained from consenting patients undergoing abdominal surgery and characterized as previously described (19). All experiments were performed with confluent HPMC from the second passage. Cells were washed and growth arrested for 48 hours in serumfree culture medium before cytokine stimulation. Growth- arrested HPMC were stimulated at $37^{\circ} \mathrm{C}$ under the conditions specified in the figure legends. Culture supernatants were harvested, centrifuged to remove cellular debris, and stored at $-70^{\circ} \mathrm{C}$ until assayed by ELISA. All data for in vitro cytokine production is expressed in picograms per $10^{5} \mathrm{HPMC}$.

Northern blot analysis. Total cellular RNA was extracted from HPMC using RNA Isolator (Genosys Biotechnologies Ltd., Poole, United Kingdom). Ten micrograms of total cellular RNA was fractionated in a formaldehyde/ $1 \%$ agarose gel. RNA was transferred overnight to nylon membrane (Boehringer-Mannheim, Lewis, United Kingdom) by capillary action and fixed by UV irradiation. Membranes were preincubated in hybridization solution $(5 \times$ SSC, $0.1 \%$ N-lauroylsarcosine, $0.02 \%$ [vol/vol] SDS, $1 \%$ [wt/vol] Boehringer-Mannheim blocking reagent) for 1 hour at $42^{\circ} \mathrm{C}$. Blots were hybridized overnight at $42^{\circ} \mathrm{C}$ with a digoxigenin (DIG) -labeled oligonucleotide probe (10 $\mathrm{ng} / \mathrm{ml}$ ) for human IL-6 (R\&D Systems). Membranes were washed and nonspecific binding sites blocked by incubating the membrane for 30 minutes in $1 \%$ (wt/vol) blocking reagent. Labeled transcripts were detected using an alkaline phosphatase-conjugated anti-DIG antibody (1:10,000 dilution; BoehringerMannheim) and visualized by autoradiography using the chemiluminescent substrate CSPD (Boehringer-Mannheim). Membranes were stripped in boiling $0.1 \%$ (wt/vol) SDS (twice for 30 minutes each) and reprobed with a DIG-labeled oligonucleotide for $\beta$-actin (10 ng/ml, R \& D Systems). Blots were developed as described above. Autoradiographs were quantified by densitometry and expressed as a ratio of the housekeeping gene $\beta$-actin.

Analysis of nuclear extracts. Nuclear extracts were prepared from HPMC using a rapid technique for the extraction of DNA binding proteins. Briefly, cells were harvested in ice-cold PBS ( $\mathrm{pH}$ 7.4) and pelleted by centrifugation. Cells were resuspended in ice-cold buffer A (10 mm HEPES-KOH [pH 7.9], $1.5 \mathrm{mM} \mathrm{MgCl} 2,10$ $\mathrm{mM} \mathrm{KCl}, 0.5 \mathrm{mM}$ DTT, $0.2 \mathrm{mM}$ PMSF) and incubated on ice for 10 minutes. The cell pellet was collected by centrifugation, resuspended in buffer B $(20 \mathrm{mM}$ HEPES-KOH [pH 7.9], 25\% glycerol, $420 \mathrm{mM} \mathrm{NaCl}, 1.5$ $\mathrm{mM} \mathrm{MgCl} 2,0.2 \mathrm{mM}$ EDTA, $0.3 \mathrm{mM}$ DTT, $0.2 \mathrm{mM}$ PMSF), and incubated on ice for 20 minutes to allow for high salt extraction. Cellular debris was removed by brief high-speed centrifugation (1200 $g$ for 10 seconds at $4^{\circ} \mathrm{C}$ ), and the resulting supernatants (nuclear extract) were collected. Protein concentrations were 
determined using the Bradford method. Electrophoretic mobility shift assays (EMSA) were performed as previously described (20). Oligonucleotides containing consensus motifs for NF- $\mathrm{KB}\left(5^{\prime}\right.$-gaTCCATGGGGAATTCCCC-3' and $3^{\prime}$-AGGTACCCCTTAAGGGGag-5') were annealed for use in EMSA. These double-stranded fragments were labeled with $\left[\alpha^{32} \mathrm{P}\right] \mathrm{dTTP}$ (Amersham-Pharmacia Biotech, Amersham, United Kingdom) using the Klenow fragment of DNA polymerase I. The composition of protein/DNA complexes was determined by supershift assays using rabbit poly-

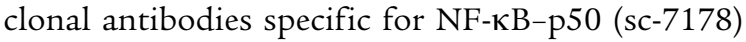
and NF-KB-p65 (sc-109) (Santa Cruz Biotech Inc., Santa Cruz, California, USA).

Mouse strains. All experimental procedures were carried out under Home Office project license 40/2131. Inbred wild-type C57/BL6 mice were purchased from Charles River UK Ltd (Margate, United Kingdom). IL-6deficient $\left(I L-6^{-/-}\right)$and IFN- $\gamma$-deficient $\left(I F N-\gamma^{\prime-}\right)$ mice, both on the C57/BL6 background, were bred in house from breeding pairs originally purchased from the Jackson Laboratory (Bar Harbor, Maine, USA) $(21,22)$. All mice were maintained under barrier conditions and were pathogen free as assessed by regular microbiologi$\mathrm{cal}$ screening. Mice were between 6 and 12 weeks of age and were weight matched for each experiment.

Preparation of S. epidermidis cell-free supernatant and definition of biological activity. S. epidermidis cell-free supernatant (SES) was prepared as previously described (16, 23). Supernatants were free of live bacteria, as assessed by lack of growth on diagnostic sensitivity testing agar. Lyophilized stocks of SES were stored at $-70^{\circ} \mathrm{C}$. The biological activity of SES was determined by monitoring IL-6 secretion from RAW 264 mouse macrophages (European Collection of Cell Cultures, Salisbury, Wiltshire, United Kingdom). RAW 264 cells were maintained in 10\% FCS/DMEM F12 MIX supplemented with $100 \mathrm{U} / \mathrm{ml}$ penicillin, $100 \mathrm{U} / \mathrm{ml}$ streptomycin, 2 $\mathrm{mM}$ L-glutamine, and a 1:100 dilution of nonessential amino acids at $37^{\circ} \mathrm{C} / 5 \% \mathrm{CO}_{2}$ in a humidified atmosphere. For bioassay, RAW 264 cells were adhered to $24-$ well plates at a density of $7.5 \times 10^{4}$ cells per well and stimulated with serial dilutions of lyophilized SES. After 24 hours, supernatants were collected and IL- 6 was quantified. For experimental reproducibility, SES fractions (equivalent to $5 \times 10^{4} \mathrm{CFU} / \mathrm{ml}$ ) were only used when IL- 6 secretion by RAW 264 exceeded 300 pg per $10^{4}$ cells.

Induction of acute peritoneal inflammation. Peritoneal inflammation was induced in vivo by administration of SES or recombinant murine IL-1 $\beta$. Groups of wildtype, $I F N-\gamma^{\prime-}$, and $I L^{-6^{-/-}}$mice received $500 \mu \mathrm{l}$ of SES, IL- $1 \beta$, or PBS through the intraperitoneal route. At defined time points, animals were sacrificed, and their peritoneum was lavaged with $2 \mathrm{ml}$ of ice-cold PBS. The composition of the leukocyte infiltrate was assessed using a Coulter counter (Coulter Z2, Beckman-Coulter UK Ltd., High Wycombe, United Kingdom) and by differential cell staining. Lavage fluids were rendered cell free by centrifugation for analysis of inflammatory mediators. To modulate the inflammatory response, wild-type, IFN- $\gamma^{-/}$, or $I L-6^{-/-}$mice were treated intraperitoneally with $500 \mu \mathrm{l}$ of SES, IL-1 $\beta$, (100 ng per mouse), IFN- $\gamma$ (0.05-50 U per mouse), HYPER-IL-6 (40 ng per mouse), and sgp130 (150 ng per mouse) as indicated in the figure legends. PBS was used as a control. At intervals after administration, mice were sacrificed and lavage parameters assessed.

Cytokine and chemokine production. Murine IL-6, KC, and IL-1 $\beta$ levels were quantified using commercially available ELISA kits (R\&D Systems). Human IL-6 was quantified using matched antibody pairs from $R \& D$ Systems in an ELISA (24).

Assessment of apoptosis in vivo (annexin V/propidium iodide staining). At defined intervals after SES stimulation, mice were sacrificed and the peritoneal cavity lavaged. Total leukocytes were recovered, and apoptosis was determined by double staining with annexin $V$ and propidium iodide (PI) according to the protocol instructions (R\&D Systems). The proportion of apoptotic leukocytes was assessed using a Becton-Dickinson FACSCalibur flow cytometer (Becton-Dickinson, Oxford, United Kingdom) using defined forward- and side-scatter settings for mouse leukocytes. Analysis of annexin $V$ staining was performed on gated neutrophils, and the quadrants were aligned in accordance with autofluorescent controls. Ten thousand gated events were acquired for each sample.

Assessment of apoptosis in vivo (caspase 3 activity). After SES stimulation, mice were sacrificed and total leukocytes recovered from the peritoneal cavity by lavage. Caspase 3 activity was assessed in cell pellets after lysis according to the manufacturer's instructions (Apoalert, BD-Clontech UK, Basingstoke, United Kingdom). Briefly, caspase 3 activity was assessed by cleavage of $\mathrm{p}$-nitroaniline (pNA) from a caspase-specific substrate DEVD-pNA at $37^{\circ} \mathrm{C}$ for 60 minutes and expressed as nanomoles of pNA per $10^{6}$ leukocytes. To confirm specificity of the observed changes, an inhibitor of caspase 3 activity (DEVD-fmk) was added to parallel samples of activated leukocytes isolated from wild-type mice injected with SES.

To modulate sIL-6R signaling in these experiments, mice were intraperitoneally administered with SES in combination with HYPER-IL-6 (40 ng per mouse) or sgp130 (150 ng per mouse), as indicated in the figure legends, before assessment of apoptosis.

Statistical comparisons. Data are expressed as means \pm SEM, and statistical analysis was performed using a Student's unpaired $t$ test (Statview SE+Graphics [Version 1.03] software). A $P$ value of less than 0.05 was considered statistically significant.

\section{Results}

IFN- $\gamma$ deficiency disrupts PMN infiltration during peritoneal inflammation. We have previously observed that IFN- $\gamma$ differentially controls ELR ${ }^{+}$CXC and CC chemokine expression by HPMC (8). To establish the overall effect of IFN- $\gamma$ on leukocyte recruitment, peritoneal 

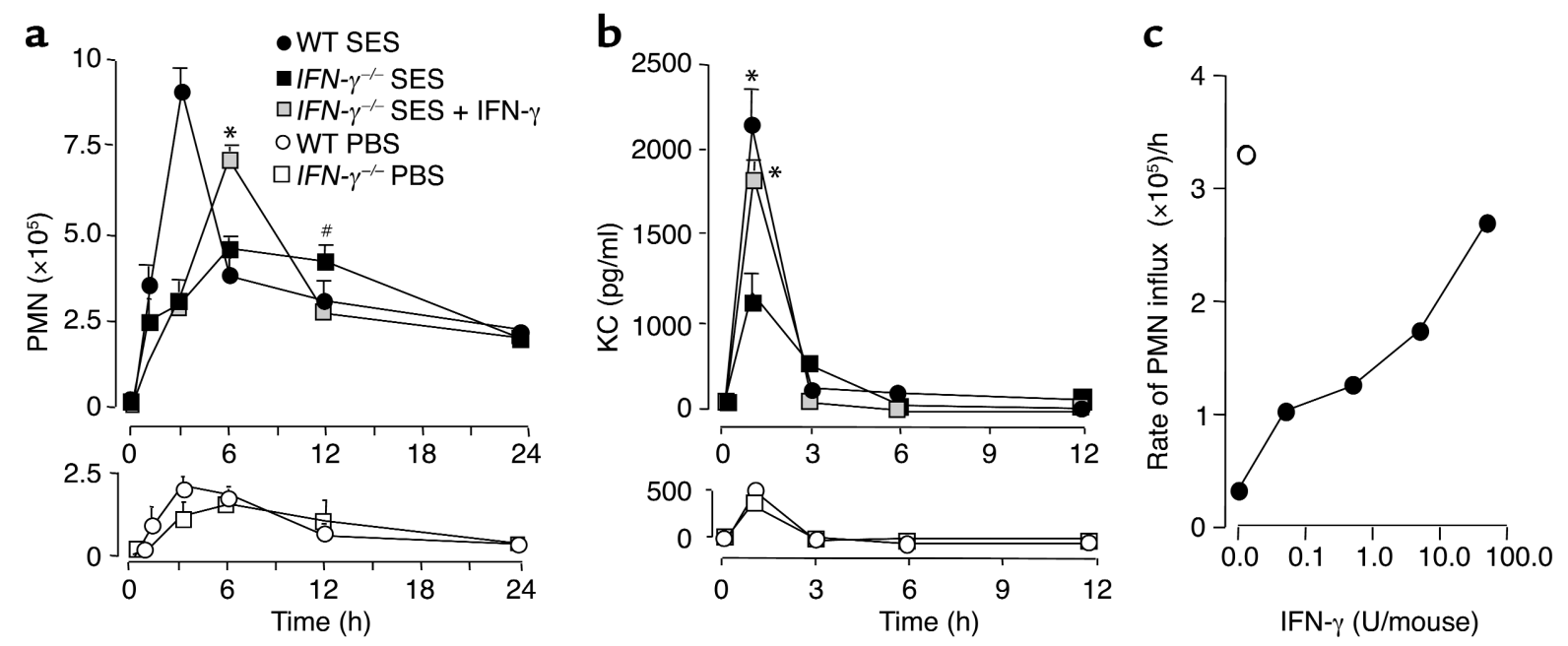

\section{Figure 1}

IFN- $\gamma$ deficiency modulates the PMN infiltration in SES-induced peritoneal inflammation. (a) Wild-type and IFN- $\gamma^{-/}$mice were intraperitoneally administered with PBS, SES, or SES in combination with IFN- $\gamma$ ( $50 \mathrm{U}$ per mouse). At defined time points, PMN infiltration was assessed using differential cell counting ( ${ }^{*} P<0.05$, a significant increase versus IFN- $\gamma^{-/}$plus $\mathrm{SES}$; $\# P<0.05$, a significant decrease versus IFN- $\gamma^{\prime-}$ plus SES). (b) KC levels were quantified by ELISA ( ${ }^{*} P<0.05$, a significant increase versus IFN- $\gamma^{\prime-}$ plus SES). Results are expressed as the mean \pm SEM of 10 mice per treatment. (c) The rate of PMN infiltration in response to exogenous administration of IFN- $\gamma(0.5-500$ $\mathrm{U}$ per mouse) to IFN- $\gamma^{-/-}$mice. Filled circles represent IFN- $\gamma^{-/-}$mice receiving SES in combination with defined doses of IFN- $\gamma$. The open circle illustrates the rate of PMN infiltration in wild-type mice.

inflammation was induced in wild-type and $I F N-\gamma^{-1}$ mice by administration of SES (16). In wild-type mice, this resulted in a time-dependent influx of PMN into the peritoneal cavity, which peaked at 3 hours (Figure 1a). In the absence of IFN- $\gamma$, fewer infiltrating PMN were detected (Figure 1a). Indeed, the rate of PMN recruitment in $I F N-\gamma^{/-}$mice was impaired, and PMN numbers remained elevated for up to 12 hours after induction of inflammation (Figure 1a). Consistent with these observations, aberrant PMN recruitment in $I F N-\gamma^{-/}$mice was also accompanied by a defect in KC production (Figure $1 \mathrm{~b}$ ). Thus, IFN- $\gamma$ might affect both the initial PMN recruitment phase and their subsequent clearance.

IFN- $\gamma$ regulates the initial rate of PMN infiltration. Reconstitution of IFN- $\gamma$ signaling in $I F N-\gamma /-$ mice facilitated a dose-dependent increase in the initial rate of
PMN infiltration (Figure 1c), with $50 \mathrm{U}$ of IFN- $\gamma$ per mouse restoring the profile of intraperitoneal $\mathrm{KC}$ secretion to that observed in wild-type mice (Figure 1b). However, it is evident that other inflammatory processes must also affect PMN infiltration, since maximal PMN recruitment was not encountered until approximately 6 hours after reconstitution with $50 \mathrm{U}$ of IFN- $\gamma$ per mouse (Figure 1a).

IFN- $\gamma$ deficiency is associated with a defect in IL-1 $\beta$ and IL-6 production. Administration of SES induced an early increase in intraperitoneal IL- $1 \beta$ production that peaked at 1 hour in wild-type mice (Figure 2a). However, this profile of IL-1 $\beta$ was altered in $I F N-\gamma^{-}$ mice and resulted in both reduced levels and a delay in the peak of IL-1 $\beta$ production (Figure $2 a$ ). Thus, the effect of IFN- $\gamma$ on PMN recruitment may occur as a consequence of this impaired expression of IL-1 $\beta$. We
Figure 2

IL-1 $\beta$ and IL-6 production after SES-induced peritoneal inflammation in wild-type and IFN- $\gamma^{/-}$ mice. (a) Wild-type or IFN- $\gamma^{-1-}$ mice were intraperitoneally administered with PBS or SES. At specific time intervals, mice were sacrificed, and intraperitoneal IL- $1 \beta$ levels were quantified using ELISA ( ${ }^{*} P<0.05$ versus PBS). (b) Wild-type or IFN- $\gamma^{-/}$mice were intraperitoneally administered with PBS, SES, IFN- $\gamma$ ( $0.5 \mathrm{U}$ per mouse), or a combination of SES and IFN- $\gamma$. Intraperitoneal IL-6 levels were quantified at defined time intervals $\left({ }^{*} P<0.05\right.$ versus IFN $-\gamma^{-1-}$ plus SES alone). The lower panels show wild-type and IFN- $\gamma^{-/}$mice treated with PBS alone. Values represent the mean \pm $\operatorname{SEM}(n=6$ mice per condition).

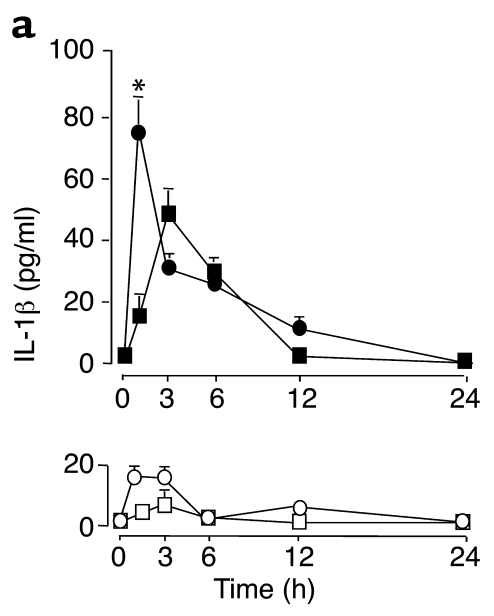



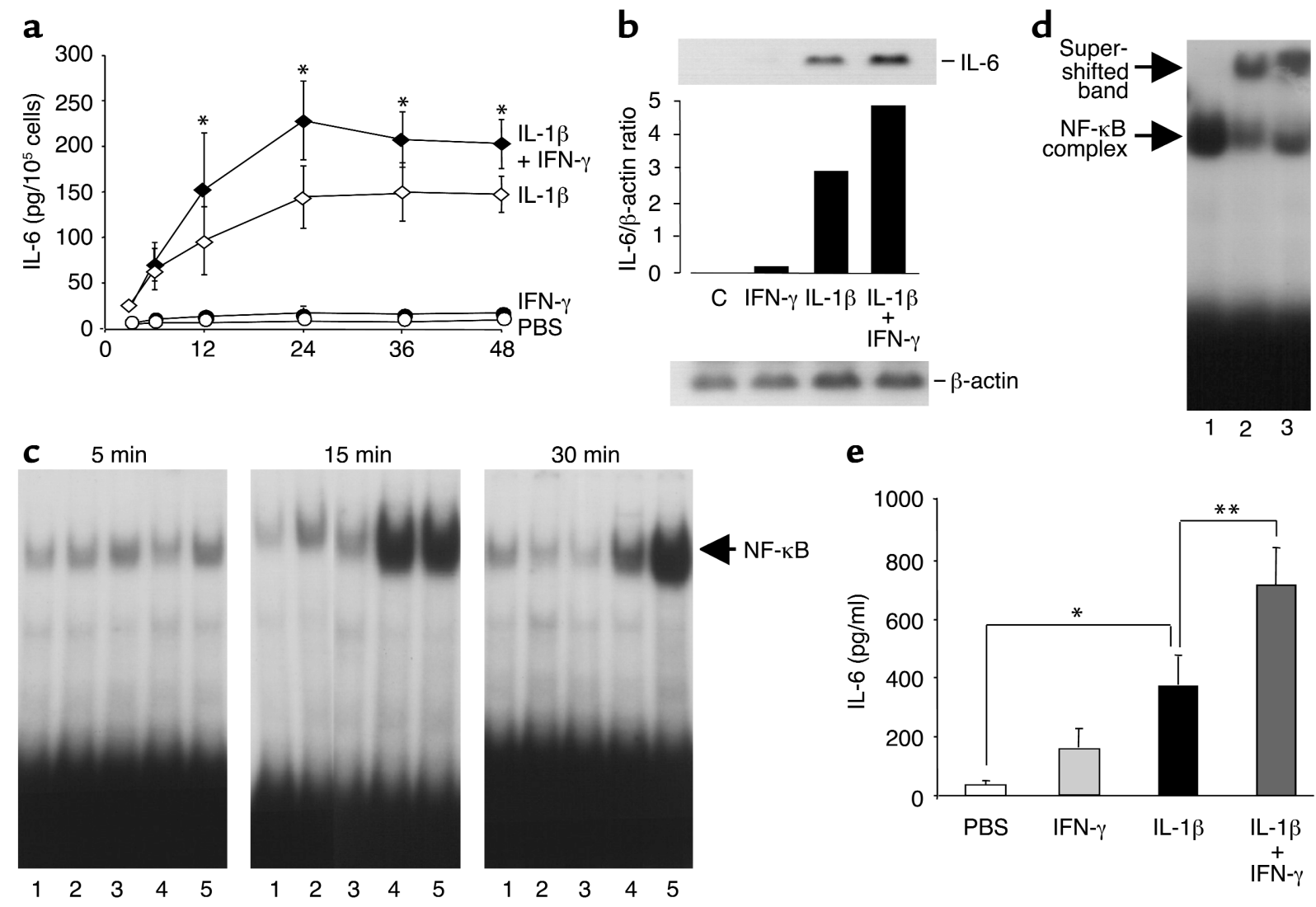

Figure 3

Regulation of IL- 6 production by IL- $1 \beta$ and IFN- $\gamma$ in vitro and in vivo. Growth-arrested HPMC were (a) incubated with medium alone or $\mathrm{IL}-1 \beta(100 \mathrm{pg} / \mathrm{ml})$ in the presence or absence of IFN $-\gamma(100 \mathrm{U} / \mathrm{ml})$. At specific time points, IL-6 levels were quantified using ELISA $\left({ }^{*} P<0.05\right.$, a significant increase versus the additive value of IL-1 $\beta$ plus IFN- $\gamma$ alone). (b) Northern blot analysis of total RNA isolated from HPMC incubated for 3 hours with medium and IL- $1 \beta(100 \mathrm{pg} / \mathrm{ml})$ in the presence or absence of IFN- $\gamma(100 \mathrm{U} / \mathrm{ml})$. Representative results for three separate experiments are shown. (c) HPMC were stimulated with medium alone (lane 1), HYPER-IL-6 (500 pg/ml, lane 2), IFN- $\gamma(100 \mathrm{U} / \mathrm{ml}$, lane 3), IL-1 $\beta$ (100 pg/ml, lane 4), or a combination of IL-1 $\beta$ and IFN- $\gamma$ (lane 5). At the indicated intervals, nuclear extracts were prepared

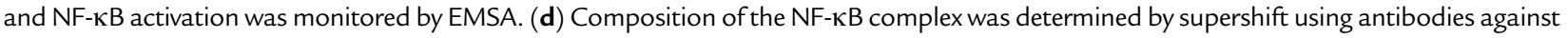
p50 (lane 2) and p65 (lane 3). No antibodies were included in lane 1. Results are representative of three separate experiments performed with HPMC from different donors. (e) Wild-type mice were intraperitoneally administered with PBS, IL- $1 \beta$ ( 100 ng per mouse), IFN- $\gamma(0.5 U$ per mouse), or a combination of IL- $1 \beta$ and IFN- $\gamma$ at these doses. After 1 hour, mice were sacrificed and IL- 6 was quantified $\left({ }^{*} P<0.05\right.$ versus PBS alone; ${ }^{*} P<0.05$, a significant increase versus the additive value of IL- $1 \beta$ plus IFN- $\gamma$ alone).

have established that IL-6, in combination with sIL-6R, regulates the transition between PMN and MNC infiltration in a similar manner to that elicited by IFN- $\gamma(8,16)$. To determine whether intraperitoneal IL- 6 levels are influenced by IFN- $\gamma$, inflammation was provoked in both wild-type and $I F N-\gamma^{/-}$mice with SES. Administration of SES to wild-type mice resulted in a time-dependent increase in IL- 6 generation, which peaked at 1 hour (Figure $2 b$ ). However, the profile of IL-6 production in IFN- $\gamma^{-/}$mice was significantly attenuated (threefold lower at 1 hour) but was almost completely restored to wild type by repletion with IFN- $\gamma$ (Figure $2 b$ ), suggesting that IFN- $\gamma$ locally regulates intraperitoneal IL-6 levels.

IFN- $\gamma$ regulates the secretion of IL-6 by mesothelial cells. We have previously demonstrated that mesothelial cells are the major producers of IL- 6 within the peritoneal cavity $(24,25)$, and its production plays a central role in regulating leukocyte recruitment
(16). Exposure of HPMC to IL- $1 \beta$ resulted in a timeand dose-dependent increase in IL- 6 production, which was synergistically enhanced by the simultaneous presence of IFN- $\gamma$ (Figure 3a). Synergistic augmentation of the IL- $1 \beta$ response by IFN- $\gamma$ was confirmed at the transcriptional level by Northern blot analysis (Figure 3b).

To establish if the synergistic effects of IFN- $\gamma$ on IL-1 $\beta$-driven IL- 6 production occurs at the level of their signal transduction pathways, activation of the Jak-STAT and NF-KB pathways was monitored by EMSA. STAT1 activation was maximal after $30 \mathrm{~min}-$ utes of stimulation with IFN- $\gamma$ alone and was unaffected by the presence of IL- $1 \beta$ (data not shown). In contrast, activation of NF- $\kappa$ B by IL- $1 \beta$ alone was maximal after 15 minutes (Figure $3 \mathrm{c}$ ), with the intensity of the banding declining thereafter. However, in combination with IFN- $\gamma$, the IL- $1 \beta$-induced activation of NF- $\kappa \mathrm{B}$ was maintained over the whole time course 

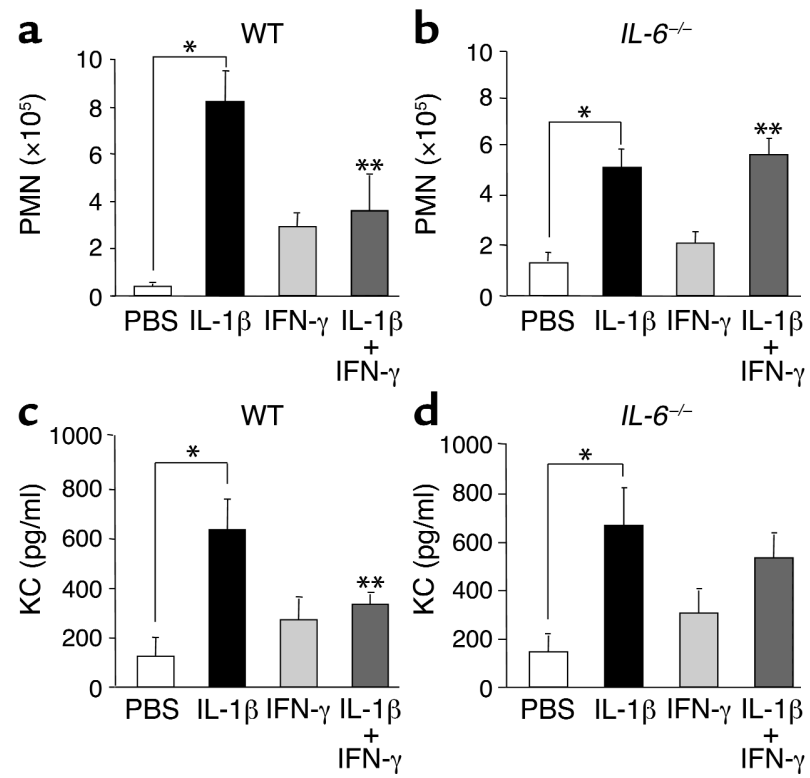

\section{Figure 4}

Regulation of PMN clearance by IFN- $\gamma$ is dependent on IL- 6 activity. Wild-type (a and $\mathbf{c}$ ) and $I L-6^{-/-}$(b and $\mathbf{d}$ ) mice were intraperitoneally administered with PBS, IL-1 $\beta$ (100 ng per mouse), IFN- $\gamma(0.5 \mathrm{U}$ per mouse), or IFN- $\gamma$ in combination with IL-1 $\beta$ at these doses. PMN infiltration was assessed at 3 hours using differential cell counting ( $\mathbf{a}$ and $\mathbf{b}$ ), and intraperitoneal KC levels were quantified at 1 hour by ELISA ( $c$ and $\mathbf{d})$. Results are expressed as the mean $\pm \operatorname{SEM}(n=5$ mice per condition; ${ }^{*} P<0.05$ versus PBS; ${ }^{*} P<0.05$, a significant decrease versus IL-1 $\beta$ alone).

studied (Figure 3c). Supershift analysis identified that the NF- $\mathrm{KB}$ complex resulted from activation of the p50 and p65 NF- $\mathrm{KB}$ subunits (Figure 3d).

IFN- $\gamma$ regulates intraperitoneal IL-6 production in vivo. To confirm the observations presented in Figure 3 ( $a$ and $b$ ), IL- 6 production by IFN- $\gamma$ was monitored in vivo. Inflammation was induced in wild-type mice using IL- $1 \beta$ as an inflammatory stimulus (Figure 3e). Although administration of IFN- $\gamma$ alone had no significant effect on intraperitoneal IL- 6 secretion, coadministration with IL-1 $\beta$ (100 ng per mouse) resulted in a synergistic increase in intraperitoneal IL-6 levels (Figure 3e).

IFN- $\gamma$ controls PMN recruitment in wild-type but not in $I L-6^{-/}$mice. In previous studies, we have demonstrated the ability of IFN- $\gamma$ to downregulate IL- $1 \beta$-induced $\mathrm{ELR}^{+} \mathrm{CXC}$ chemokine production and PMN recruitment into the peritoneal cavity of wild-type mice (8). To investigate if IFN- $\gamma$ downregulates PMN recruitment

\section{Figure 5}

Reconstitution of IL-6/sIL-6R signaling in IFN- $\gamma^{-/-}$mice normalizes the control of PMN clearance. IFN- $\gamma^{-1}$ mice were intraperitoneally administered with PBS, SES, IL-1 $\beta$ (100 pg/ml), HYPER-IL-6 (40 ng per mouse, $\mathrm{H}-\mathrm{IL}-6)$, or combinations of SES with HYPER-IL-6 or IL-1 $\beta$ with HYPER-IL-6. PMN infiltration was assessed at 3 hours through differential cell counting ( $\mathbf{a}$ and $\mathbf{c}$ ), and intraperitoneal KC levels were quantified at 1 hour ( $\mathbf{b}$ and $\mathbf{d})$ ). Results are expressed as the mean $\pm \operatorname{SEM}\left(n=5\right.$ mice per condition; ${ }^{*} P<0.05$ versus PBS; ${ }^{*} P<0.05$, a significant decrease versus SES or IL-1 $\beta$ alone).

through an effect on IL-6 signaling, we examined the ability of IFN- $\gamma$ to control PMN recruitment in wildtype and $I L-6^{-/-}$mice. In wild-type mice, IL-1 $\beta$-induced PMN recruitment and KC production were both significantly reduced after IFN- $\gamma$ treatment (Figure 4, a and c). However, intraperitoneal administration of IFN- $\gamma$ to $I L-6^{-/-}$mice did not alter PMN recruitment or KC production (Figure $4, \mathrm{~b}$ and $\mathrm{d}$ ).

IL-6 signaling restores the control of PMN trafficking in IFN- $\gamma$ deficiency. Data in $I L-6^{-/-}$mice indicated that the ability of IFN- $\gamma$ to control PMN recruitment is coupled to the in vivo expression of IL-6. To confirm this dependency on IL-6, inflammation was induced in $I F N-\gamma^{-/}$mice with either SES or IL-1 $\beta$. To reconstitute IL-6 signaling, mice were administered with an engineered IL-6/sIL-6R fusion protein (HYPER-IL-6) (18) in combination with the activators of inflammation (SES or IL-1 $\beta$ ). HYPER-IL-6 has previously been shown to suppress PMN infiltration (16) and, as predicted, significantly suppressed KC production and PMN recruitment in $I F N-\gamma^{/-}$mice in response to either SES- or IL-1 $\beta$-induced activation (Figure 5).

IFN- $\gamma$ modulates PMN apoptosis through regulation of IL-6 activity. By a comparison of the profile of PMN infiltration in wild-type and $I F N-\gamma^{-/}$mice, it appears that IFN- $\gamma$ directly influences the initial phase of recruitment of PMN and also indirectly acts to control their subsequent clearance (Figure 1a). Using annexin V/PI staining activity as a marker of apoptosis, we observed that PMN apoptosis after SES activation was defective in the absence of IFN- $\gamma$ and remained impaired throughout the duration of the clearance phase (Table 1). Indeed, by comparing the profiles of the annexin V/PI scatter plots, it is evident that the proportion of both early (lower right quadrant) and late apoptotic/necrotic (upper right quadrant) PMN was significantly impaired in both $I F N-\gamma^{--}$and $I L-6^{-/-}$mice

a
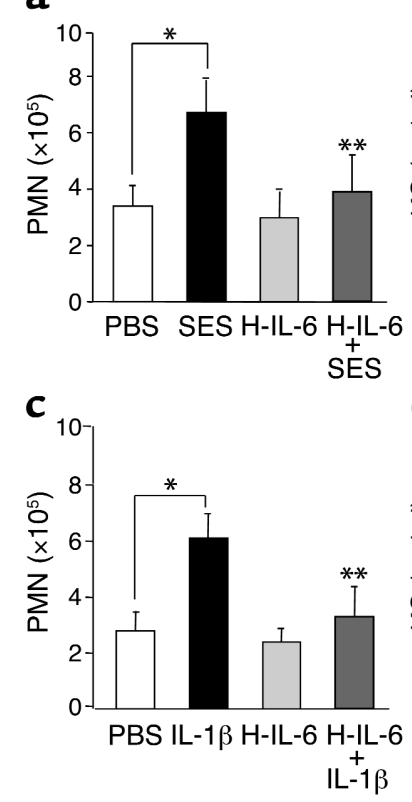

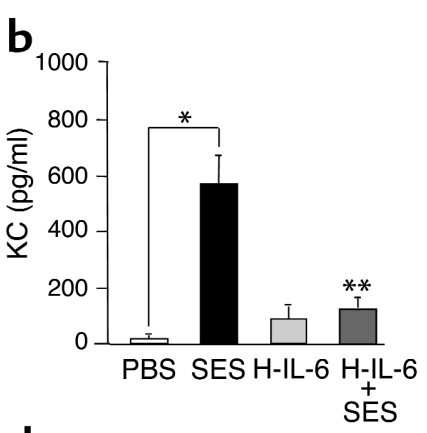

d

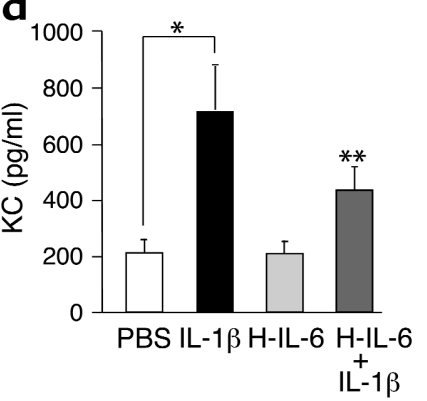


Table 1

Profile of intraperitoneal apoptosis in wild-type and $I F N-\gamma^{-1}$ mice

\begin{tabular}{|c|c|c|c|c|}
\hline \multirow{3}{*}{$\begin{array}{l}\text { Time } \\
\text { (hours) }\end{array}$} & \multicolumn{4}{|c|}{ Apoptotic leukocytes (\%) } \\
\hline & \multicolumn{2}{|c|}{ Wild-type mice } & \multicolumn{2}{|c|}{$I F N-\gamma^{-/}$mice } \\
\hline & LR & UR & LR & UR \\
\hline 6 & $14.2 \pm 1.75$ & $13.2 \pm 4.4$ & $8.50 \pm 1.20^{\mathrm{A}}$ & $5.9 \pm 0.9^{A}$ \\
\hline 9 & $13.1 \pm 0.40$ & $9.4 \pm 0.4$ & $9.79 \pm 1.50^{\mathrm{A}}$ & $6.9 \pm 1.26$ \\
\hline 12 & $17.2 \pm 1.75$ & $9.26 \pm 0.7$ & $13.19 \pm 1.98^{\mathrm{A}}$ & $8.2 \pm 0.3^{\mathrm{A}}$ \\
\hline
\end{tabular}

Apoptotic leukocytes elicited from SES-stimulated mice were monitored by FACS analysis using annexin V/PI dual labeling. Data are taken from the lower right (LR) and upper right (UR) quadrants to represent the proportion of early apoptotic and late apoptotic/necrotic leukocytes. Values represent the mean $\pm \operatorname{SEM}\left(n=4-6\right.$ mice per condition, ${ }^{A} P<0.05$ versus respective quadrant value).

(Figure 6). Defective PMN apoptosis in $I F N-\gamma^{/-}$and $I L-6^{-/-}$mice was also confirmed by measurement of leukocyte caspase 3 activity after induction of SESinduced inflammation. As shown in Figure $7 \mathrm{~b}$, caspase 3 activity was significantly reduced in both $I F N-\gamma^{/-}$ and $I L-6^{-/-}$mice as compared with wild-type mice.

To establish a potential link between IFN- $\gamma$ and IL-6 in controlling PMN apoptosis, IL-6/sIL-6R signaling was modulated in wild-type and $I F N-\gamma^{--}$mice (Figure 7 , $a$ and b). Blockade of sIL-6R signaling in wild-type mice using sgp130 (26) significantly reduced the proportion of early apoptosis as assessed by annexin $V$ staining (lower right quadrant) but had no apparent effect on late apoptotic/necrotic PMN (Figure 7a). Under identical conditions, however, administration of sgp130 to wildtype mice significantly reduced caspase 3 activation, confirming that there was indeed a defect in apoptosis under conditions of IL-6/sIL-6R signaling deficiency (Figure $7 \mathrm{~b}$ ). Conversely, administration of HYPER-IL-6 to IFN- $\gamma^{/-}$mice restored leukocyte apoptosis to levels comparable with those seen in wild-type mice (Figure $7 \mathrm{~b})$. Thus, IFN- $\gamma$ modulates PMN apoptosis through an effect on sIL-6Rinduced signaling within the peritoneal cavity. Consistent with the notion that sIL-6R signaling is pivotal in the controlled removal of neutrophils during acute inflammation is the observation that $I L-6^{-/-}$ mice demonstrate aberrant clearance of PMN from the peritoneal cavity after SES-induced inflammation (Figure 7c) and that inhibition of sIL-6R signaling in wild-type mice, with sgp130, results in a profile of PMN recruitment consistent with that seen in $I L-6^{-/-}$mice (Figure $7 \mathrm{c}$ ). These IL-6/sIL-6R-driven effects appear to be related to the control of neutrophil clearance, since the initial rate of PMN influx remained unaffected by sgp130 blockade.

\section{Discussion}

IFN- $\gamma$ plays a central role in governing immune cell responses and T cell polarization. However, it is becoming increasingly apparent that this cytokine also orchestrates innate immune responses $(2,4)$. Although data suggest that these effects are related to induction of genes associated with innate cellular immunity, the precise mechanisms by which this cytokine contributes to host defense and the resolution of inflammation remain to be fully identified (1). To ensure that the inflammatory response provides an efficient mechanism for eradicating bacterial infection without damaging host tissues, leukocyte recruitment, activation, and clearance is tightly regulated (17). Previously, we have established that IFN- $\gamma$ can modulate chemokine production and leukocyte recruitment in response to proinflammatory cytokines (8). Coordination of these processes is highly reminiscent of the inflammatory events controlled by IL- 6 and its soluble receptor, sIL-6R (16). In light of the congruence between the activities of IFN- $\gamma$ and IL-6, we have now explored the potential interplay between these inflammatory cytokines in regulating leukocyte trafficking.

Initial experiments examined the effect of IFN- $\gamma$ deficiency on the process of PMN recruitment. Induction of inflammation in $I F N-\gamma^{/-}$mice with SES resulted in modulation of the PMN recruitment profile, defined by a delay in the rate of initial accumulation, aberrant clearance, and a reduction in overall PMN numbers. Repletion experiments (with murine IFN- $\gamma$ ) resulted in partial restoration of recruitment of PMN, with the
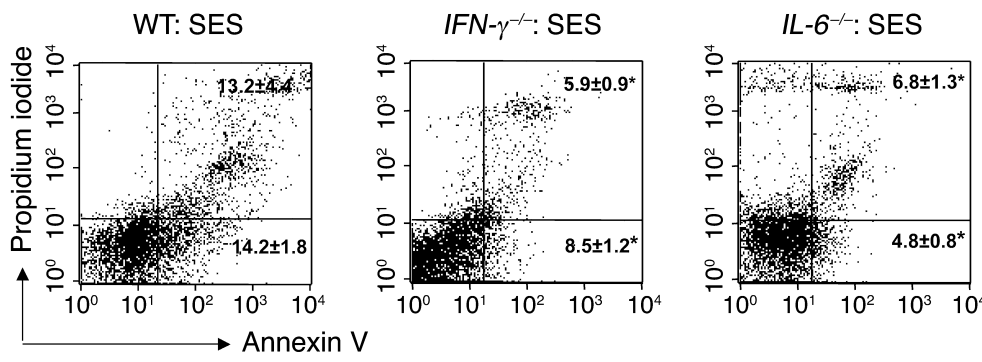

Figure 6

IFN- $\gamma$ and IL- 6 modulate leukocyte apoptosis during SES-induced peritoneal inflammation. Annexin V/PI staining of leukocytes isolated from the peritoneal cavity of wildtype, IFN- $\gamma^{/-}$, and $I L-6^{-/-}$mice. Representative scatter plots show FACS analysis of gated PMN obtained from mice stimulated with SES for 6 hours. Apoptotic cells were identified according to annexin $\mathrm{V}^{+} / \mathrm{PI}^{-}$(lower right quadrant, early apoptosis) and annexin $\mathrm{V}^{+} / \mathrm{Pl}^{+}$(upper right quadrant, late apoptosis/necrosis) staining. The proportion of cells residing in each quadrant is expressed as a percentage (mean \pm SEM, $n=4-7$ mice/condition; ${ }^{*} P<0.05$, a significant increase versus $I F N-\gamma^{-/-}$plus SES). By comparison with values from wild-type mice, mean reductions of $40.2 \%$ and $55.4 \%$ were respectively seen in early and late apoptotic events for $\mathrm{I} F \mathrm{~N}-\gamma^{-1}$ mice and of $66.2 \%$ and $48.9 \%$ for $I L-6^{-/-}$mice. The proportion of annexin $\mathrm{V}^{+} / \mathrm{PI}^{-} \mathrm{PMN}$ in mouse peripheral blood was always less than $1 \%$ (data not shown). 

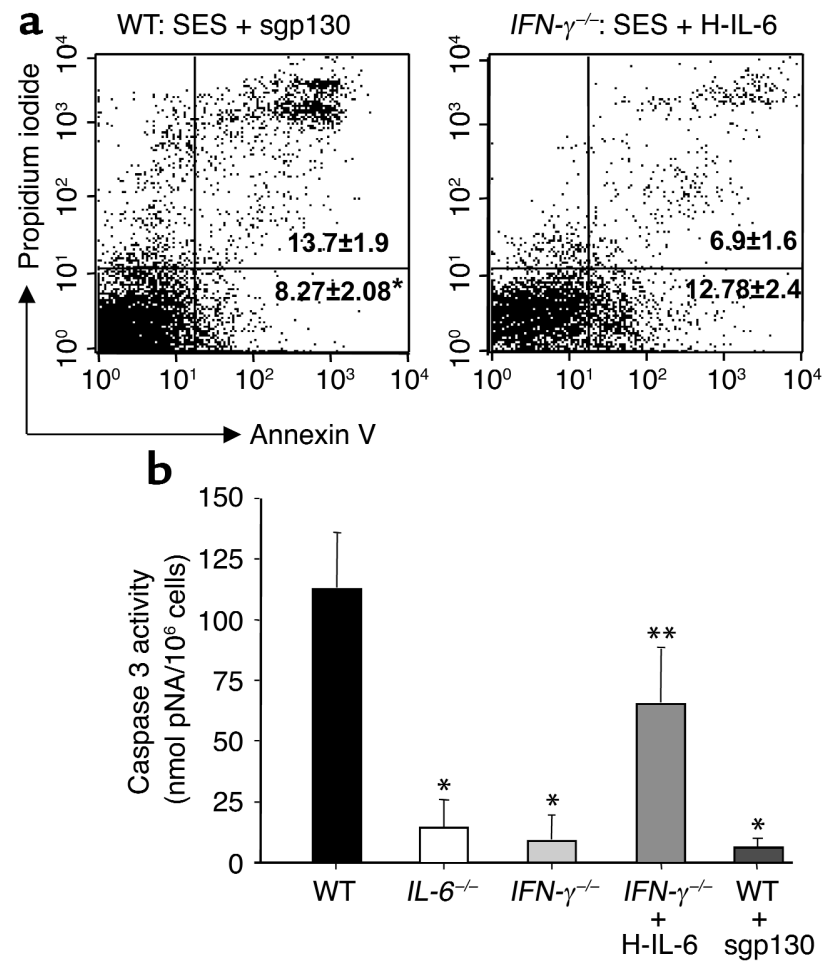

C

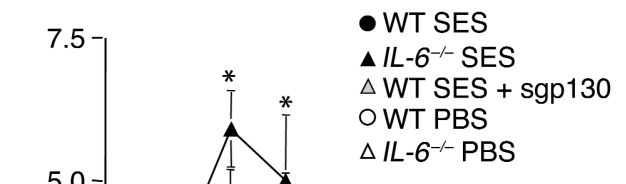

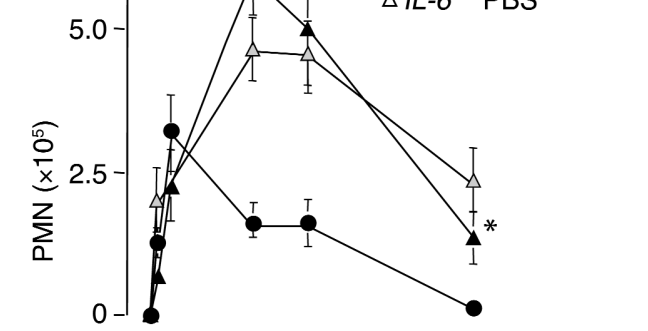

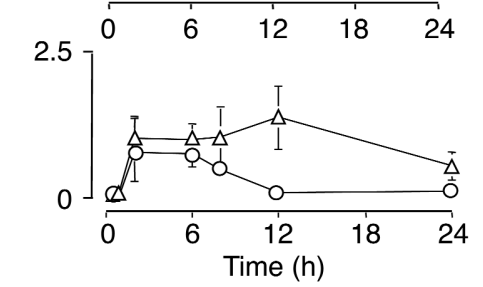

\section{Figure 7}

Modulation of IL-6 signaling in vivo affects apoptotic clearance of PMN in SES-induced peritoneal inflammation. (a) Annexin V/PI staining of leukocytes. Representative scatter plots show similar analysis performed on cells elicited from wild-type mice after 6 hours of stimulation with SES in combination with sgp130 (150 ng per mouse) or IFN- $\gamma^{-/}$mice after stimulation with SES in combination with HYPERIL-6 (40 ng per mouse). (b) Caspase 3 activity was evaluated in isolated leukocytes from wild-type, IFN- $\gamma^{-/-}$, and $I L-6^{-/-}$mice intraperitoneally administered with SES, IFN- $\gamma^{\prime-}$ mice administered with SES in combination with HYPER-IL-6 (40 ng per mouse), or wildtype mice administered with SES in combination with sgp130 (150 ng per mouse). Data (expressed as nanomoles of pNA per $10^{6}$ cells) is presented from the 6-hour time point (mean $\pm \mathrm{SEM}, n=5$ mice per condition; ${ }^{*} P<0.05$, a significant decrease versus wild type plus SES; ${ }^{*} P<0.05$, a significant decrease versus IFN- $\gamma^{\prime-}$ plus SES). Inclusion of the caspase 3 inhibitor DEVD-fmk to wild-type leukocyte extracts reduced activity to background levels (data not shown). (c) Wild-type mice were intraperitoneally administered SES, SES plus sgp130 (150 ng per mouse), or PBS, and $I L-6^{-/-}$mice were intraperitoneally administered SES or PBS. At defined intervals, PMN infiltration was assessed by differential cell count. The lower panel shows PMN influx in response to PBS alone. Results are expressed as the mean \pm SEM of 12 mice per treatment $\left({ }^{*} P<0.05\right.$ versus wild-type mice) .

most striking effect being in controlling the initial rate of their recruitment into the peritoneal cavity. IFN- $\gamma$ thus appears to directly influence the effector arm of PMN recruitment as a result of its ability to regulate the local concentrations of ELR ${ }^{+}$chemokines $(\mathrm{KC} /$ macrophage inflammatory peptide-2). This response to IFN- $\gamma$ would seem to be independent of IL- 6 , since the initial rate of PMN infiltration is unaffected by IL-6 or blockade of sIL-6R signaling with sgp130, and IL-6 deficiency is associated with greater PMN infiltration, increased $\mathrm{KC} /$ macrophage inflammatory peptide- 2 expression, and a delay in neutrophil clearance (Figure 7c) $(16,27)$.

The mechanism by which IFN- $\gamma$ controls initial PMN recruitment is at least in part related to the early secretion of IL- $1 \beta$ in response to SES, since its production is reduced after induction of inflammation in ${\mathrm{IFN}-\gamma^{-/}}^{-}$ mice. Consequently, combined IFN- $\gamma$ and IL- $1 \beta$ signaling appear to play a pivotal role in controlling the initial rate of PMN recruitment through their regulation of $\mathrm{ELR}^{+} \mathrm{CXC}$ chemokine expression. In this respect, we have previously observed that IFN- $\gamma$ dose-dependently modulates IL-1 $\beta$ - or TNF- $\alpha$-driven CXCL8 (IL-8) production (8). In the context of the peritoneal cavity, the mesothelium performs a major role in host defense against bacterial infection, and as in other organ systems, local chemokine secretion plays a pivotal role in controlling these processes $(28,29)$. Such concentration-dependent effects of IFN- $\gamma$ are relevant to bacterial peritonitis, in which temporal changes in intraperitoneal IL-1, TNF- $\alpha$, and IFN- $\gamma$ levels are observed during the progression of inflammation (12).

We have previously identified that both IFN- $\gamma$ and IL-6 signaling through sIL-6R contribute to the process of PMN removal from the peritoneal cavity and a shift from innate to acquired immunity (16). The data presented herein emphasize that a deficiency in IFN- $\gamma$ is accompanied by a defect in IL- 6 expression and PMN removal from the peritoneal cavity. Reconstitution of IFN- $\gamma$ signaling in $I F N-\gamma^{-/}$mice restored IL- 6 secretion and identifies a direct role for IFN- $\gamma$ in controlling local IL- 6 generation. Indeed, by 
modulating the activities of IL-6 (through its soluble receptor) and IFN- $\gamma$ in IL- 6 - and IFN- $\gamma$-deficient mice, we have clearly shown that IFN- $\gamma$ promotes the clearance of PMN by regulating local IL-6 levels. In vitro studies emphasize that IFN- $\gamma$ and IL- $1 \beta$ synergistically induce the expression of IL-6, paralleling studies in human monocytes (30). This interplay of IL-1 $\beta$ and IFN- $\gamma$ signaling appears to result in the maintenance of NF- $\kappa B$ activity (Figure $3 c)(31,32)$.

The apparent defect in PMN clearance in $I F N-\gamma^{/-}$ mice challenged with SES led us to examine the mechanisms controlling the apoptosis of senescent PMN. Using both annexin V/PI staining and caspase 3 activation to characterize both early and late apoptotic events (33), we identified significantly reduced levels of apoptosis in both IFN- $\gamma$ and IL- 6 deficiency. Reconstitution of IL-6/sIL-6R signaling in $I F N-\gamma^{-/}$and $\mathrm{IL}^{-6^{-/}}$ mice restored apoptosis, which emphasizes that IL-6 signaling through its soluble receptor promotes PMN apoptosis. This conclusion is directly supported by the studies presented using sgp130 as the natural sIL-6R antagonist (26). Administration of sgp130 to wild-type mice significantly blocked the in vivo activation of caspase 3 and reduced the proportion of PMN entering early apoptosis. Although in these experiments no significant difference was observed in the proportion of late apoptotic/necrotic PMN, as assessed by annexin $\mathrm{V}^{+} / \mathrm{PI}^{+}$cells, this is perhaps not surprising given that sgp130 augments PMN recruitment and residence time, while concomitantly blocking MNC infiltration (Figure 7c) (16). Such a disproportionate skewing of the leukocyte influx may ultimately impinge on the nonphlogistic removal of PMN by infiltrating MNC and result in a higher proportion of late apoptotic and necrotic cells being retained at the inflammatory focus. Although the precise cellular mechanisms governing these effects on apoptosis are yet to be defined, previous studies have identified that IFN- $\gamma$ can modulate apoptotic pathways through cathepsin D- or Fas-related pathways (34-36), whereas IL-6 can rescue T cells from apoptosis through induction of bcl-2 and bcl-xl (37). Indeed, both IL- 6 and STAT $1 / 3$ signaling have been implicated in protection against Fas-mediated cell death through modulating cell-surface Fas and antiapoptotic gene expression in T lymphocytes (38-40).

These observations on the role of sIL-6R in promoting PMN apoptosis during acute inflammation contrast with its ability to promote lymphocyte survival in chronic inflammatory conditions such as Crohn's disease (41). Interestingly, under these conditions elevated IL- 6 levels are paralleled by increases in IFN- $\gamma$, suggestive of potential interactions between these signaling pathways in controlling $\mathrm{T}$ cell apoptosis $(39,41)$, an observation recently substantiated in a model of $\mathrm{T}$ cell-mediated experimental hepatitis in $I F N-\gamma^{-/}$and $I L-6^{-/-}$mice (42). The ability of IL- 6 (and IFN- $\gamma$ ) signaling to balance pro- and antiapoptotic events in distinct leukocyte populations may be crucial to the transition between innate and acquired immunity. Indeed, such differential regulation of pro- and antiapoptotic signals may promote the nonphlogistic phagocytosis of senescent PMN, while protecting MNC (responsible for their removal) from entering apoptosis. Ultimately, these events appear to be essential in the controlled removal of PMN and the resolution of acute inflammation (43-45).

Although the controlled recruitment and removal of leukocytes is pivotal to effective host defense and the resolution of inflammation, their maintenance at sites of injury or infection contributes directly to disease pathogenesis (46). The current data suggest that interplay between IFN- $\gamma$ and IL-6/sIL-6R signaling contributes to the rapid recruitment and timely removal of PMN from sites of acute inflammation. In the context of host defense, these data provide a rationale at the level of leukocyte trafficking for the obligate role of IFN- $\gamma$ in controlling bacterial and viral infection and resolution of acute inflammation (1, 2, 4). In more chronic conditions such as hepatitis, inflammatory bowel disease, and arthritis, however, characterized by activated $T$ cell infiltration, the balance of STAT1 and STAT3 signaling (presumably controlled by local IL- 6 and IFN- $\gamma$ levels) contributes directly to disease progression $(41,42,47)$. Taken together, these data emphasize that irrespective of disease context, the regulated or deregulated interplay between the effects of IL- 6 and IFN- $\gamma$ are central to the control of leukocyte trafficking in inflammatory disease. Consequently, understanding the contribution of IFN- $\gamma$ and IL-6/sIL-6R signaling to leukocyte trafficking in both acute and chronic inflammatory states is essential if appropriate therapeutic strategies aimed at more effective host defense, or reducing the detrimental consequences of unresolved chronic inflammation, are to be achieved.

\section{Acknowledgments}

We are grateful to Patti Coan (Department of Microbiology, University of Alabama at Birmingham) for help and advice on the establishment of our mouse colonies. This work was supported by a Medical Research Council (MRC) Ph.D. studentship (to R.M. McLoughlin); a University of Wales College of Medicine, Division of Medicine Ph.D. studentship (to T.S. Wilkinson); and project grants from the Wellcome Trust (058297/2/ $99 / Z$ and $065961 / Z / 01 / Z$ to S.A. Jones and N. Topley) and the Arthritis Research Campaign (J0527 and J0538 to S.A. Jones, N. Topley, and A.S. Williams).

\footnotetext{
1. Decker, T., Stockinger, S., Karaghiosoff, M., Müller, M., and Kovarik, P. 2002. IFNs and STATs in innate immunity to microorganisms. J. Clin. Invest. 109:1271-1277. doi:10.1172/JCI200215770.

2. Meraz, M.A., et al. 1996. Targeted disruption of the Stat 1 gene in mice reveals unexpected physiologic specificity in the JAK-STAT signaling pathway. Cell. 84:431-442.

3. Boehm, U., Klamp, T., Groot, M., and Howard, J.C. 1997. Cellular responses to interferon-gamma. Annu. Rev. Immunol. 15:749-795.

4. Durbin, J.E., Hackenmiller, R., Simon, M.C., and Levy, D.E. 1996. Targeted disruption of the mouse Stat 1 gene results in compromised innate immunity to viral disease. Cell. 84:443-450.
} 
5. Struyf, S., et al. 1998. Synergistic induction of MCP-1 and-2 by IL-1 $\beta$ and interferons in fibroblasts and epithelial cells. J. Leukoc. Biol. 63:364-372.

6. Schnyder-Candrian, S., Strieter, R.M., Kunkel, S.L., and Walz, A. 1995. Interferon-alpha and interferon-gamma down-regulate the production of interleukin-8 and ENA-78 in human monocytes. J. Leukoc. Biol. 57:929-935.

7. Penton-Rol, G., et al. 1998. Selective inhibition of expression of the chemokine receptor CCR2 in human monocytes by IFN-gamma. J. Immunol. 160:3869-3873.

8. Robson, R.L., et al. 2001. Differential regulation of chemokine production in human peritoneal mesothelial cells: IFN-gamma controls neutrophil migration across the mesothelium in vitro and in vivo. J. Immunol. 167:1028-1038.

9. Jonjic, N., et al. 1992. Expression of adhesion molecules and chemotactic cytokines in cultured human mesothelial cells. J. Exp. Med. 176:1165-1174.

10. Bonecchi, R., et al. 1999. Up-regulation of CCR1 and CCR3 and induction of chemotaxis to CC chemokines by IFN-gamma in human neutrophils. J. Immunol. 162:474-479.

11. Witowski, J., et al. 2000. IL-17 stimulates intraperitoneal neutrophil infiltration through the release of GRO alpha chemokine from mesothelial cells. J. Immunol. 165:5814-5821.

12. Lu, Y., Hylander, B., and Brauner, A. 1996. Interleukin-10, interferon gamma, interleukin-2, and soluble interleukin-2 receptor alpha detected during peritonitis in the dialysate and serum of patients on continuous ambulatory peritoneal dialysis. Perit. Dial. Int. 16:607-612.

13. Hotchkiss, R.S., et al. 1999. Prevention of lymphocyte cell death in sepsis improves survival in mice. Proc. Natl. Acad. Sci. U. S. A. 96:14541-14546.

14. Baggiolini, M. 1998. Chemokines and leukocyte traffic. Nature. 392:565-568.

15. DiTirro, J., et al. 1998. Disruption of the cellular inflammatory response to Listeria monocytogenes infection in mice with disruptions in targeted genes. Infect. Immun. 66:2284-2289.

16. Hurst, S.M., et al. 2001. IL-6 and its soluble receptor orchestrate a temporal switch in the pattern of leukocyte recruitment seen during acute inflammation. Immunity. 14:705-714.

17. Meagher, L.C., et al. 1992. Phagocytosis of apoptotic neutrophils does not induce macrophage release of thromboxane B2. J. Leukoc. Biol. 52:269-273.

18. Fischer, M., et al. 1997. I. A bioactive designer cytokine for human hematopoietic progenitor cell expansion. Nat. Biotechnol. 15:142-145.

19. Topley, N., et al. 1994. Human peritoneal mesothelial cell prostaglandin synthesis: induction of cyclooxygenase mRNA by peritoneal macrophage derived cytokines. Kidney Int. 46:900-909.

20. Zhang, Z., Fuentes, N.L., and Fuller, G.M. 1995. Characterization of the IL- 6 responsive elements in the gamma fibrinogen gene promoter. J. Biol. Chem. 270:24287-24291.

21. Kopf, M., et al. 1994. Impaired immune and acute-phase responses in interleukin-6-deficient mice. Nature. 368:339-342.

22. Dalton, D.K., et al. 1993. Multiple defects of immune cell function in mice with disrupted interferon-gamma genes. Science. 259:1739-1742.

23. Mackenzie, R., Topley, N., Neubauer, A., Coles, G.A., and Williams, J.D. 1997. Staphylococcal exoproducts down-regulate cyclooxygenase 1 and 2 in peritoneal macrophages. J. Lab. Clin. Med. 129:23-34.

24. Witowski, J., Jorres, A., Coles, G.A., Williams, J.D., and Topley, N. 1996. Superinduction of IL- 6 synthesis in human peritoneal mesothelial cells is related to the induction and stabilization of IL- 6 mRNA. Kidney Int. 50:1212-1223.

25. Topley, N., et al. 1993. Human peritoneal mesothelial cells synthesize interleukin-6: induction by IL-1 beta and TNF alpha. Kidney Int. 43:226-233.

26. Jostock, T., et al. 2001. Soluble gp130 is the natural inhibitor of soluble interleukin- 6 receptor trans-signaling responses. Eur. J. Biochem. 268:160-167.

27. Xing, Z., et al. 1998. IL-6 is an anti-inflammatory cytokine required for controlling local and systemic acute inflammatory responses. J. Clin. Invest. 101:311-320.

28. Garcia-Ramallo, E., et al. 2002. Resident cell chemokine expression serves as the major mechanism for leukocyte recruitment during local inflammation. J. Immunol. 169:6467-6473.

29. Topley, N., et al. 1996. Activation of inflammation and leukocyte recruitment into the peritoneal cavity. Kidney Int. 56:S17-S21.

30. Sanceau, J., Kaisho, T., Hirano, T., and Wietzerbin, J. 1995. Triggering of the human interleukin- 6 gene by interferon-gamma and tumor necrosis factor-alpha in monocytic cells involves cooperation between interferon regulatory factor-1, NF kappa B, and Sp 1 transcription factors. J. Biol. Chem. 270:27920-27931.

31. Matsusaka, T., et al. 1993. Transcription factors NF-IL6 and NF-kappa B synergistically activate transcription of the inflammatory cytokines, interleukin 6 and interleukin 8. Proc. Natl. Acad. Sci. U. S. A. 90:10193-10197.

32. Ohmori, Y., Schreiber, R.D., and Hamilton, T.A. 1997. Synergy between interferon-gamma and tumor necrosis factor-alpha in transcriptional activation is mediated by cooperation between signal transducer and activator of transcription 1 and nuclear factor $\kappa$ B. J. Biol. Chem. 272:14899-14907.

33. Dong, H., et al. 2003. Costimulating aberrant T cell responses by B7$\mathrm{H} 1$ autoantibodies in rheumatoid arthritis. J. Clin. Invest. 111:363-370. doi:10.1172/JCI200316015.

34. Sobek, V., Balkow, S., Korner, H., and Simon, M.M. 2002. Antigeninduced cell death of T effector cells in vitro proceeds via the Fas pathway, requires endogenous interferon-gamma and is independent of perforin and granzymes. Eur. J. Immunol. 32:2490-2499.

35. Novelli, F., et al. 1994. Environmental signals influencing expression of the IFN-gamma receptor on human T cells control whether IFNgamma promotes proliferation or apoptosis. J. Immunol. 152:496-504.

36. Deiss, L.P., Galinka, H., Berissi, H., Cohen, O., and Kimchi, A. 1996. Cathepsin D protease mediates programmed cell death induced by interferon-gamma, Fas/APO-1 and TNF-alpha. EMBOJ. 15:3861-3870.

37. Teague, T.K., Marrack, P., Kappler, J.W., and Vella, A.T. 1997. IL-6 rescues resting mouse T cells from apoptosis. J. Immunol. 158:5791-5796.

38. Kovalovich, K., et al. 2001. Interleukin-6 protects against Fas-mediated death by establishing a critical level of anti-apoptotic hepatic proteins FLIP, Bcl-2, and Bcl-xL. J. Biol. Chem. 276:26605-26613.

39. Teague, T.K., et al. 2000. Activation-induced inhibition of interleukin 6-mediated T cell survival and signal transducer and activator of transcription 1 signaling. J. Exp. Med. 191:915-926.

40. Ivanov, V.N., et al. 2001. Cooperation between STAT3 and c-jun suppresses Fas transcription. Mol. Cell. 7:517-528.

41. Atreya, R., et al. 2000. Blockade of interleukin 6 trans signaling suppresses T-cell resistance against apoptosis in chronic intestinal inflammation: evidence in Crohn disease and experimental colitis in vivo. Nat. Med. 6:583-588.

42. Hong, F., et al. 2002. Opposing roles of STAT1 and STAT3 in T cellmediated hepatitis: regulation by SOCS. J. Clin. Invest. 110:1503-1513. doi:10.1172/JCI200215841.

43. Kaplanski, G., Marin, V., Montero-Julian, F., Mantovani, A., and Farnarier, C. 2003. IL-6: a regulator of the transition from neutrophil to monocyte recruitment during inflammation. Trends Immunol. 24:25-29.

44. Savill, J. 1997. Apoptosis in resolution of inflammation. J. Leukoc. Biol. 61:375-380.

45. Savill, J., Dransfield, I., Gregory, C., and Haslett, C. 2002. A blast from the past: clearance of apoptotic cells regulates immune responses. Nat. Rev. Immunol. 2:965-975.

46. Dibbert, B., et al. 1999. Cytokine-mediated Bax deficiency and consequent delayed neutrophil apoptosis: a general mechanism to accumulate effector cells in inflammation. Proc. Natl. Acad. Sci. U. S. A. 96:13330-13335.

47. Atsumi, T., et al. 2002. A point mutation of Tyr-759 in interleukin 6 family cytokine receptor subunit gp130 causes autoimmune arthritis. J. Exp. Med. 196:979-990. 